\title{
PERSISTENACIA BIOLOGICA DE AMETRYN, DIURON E OXYFLUORFEN NO SOLO
}

\author{
Biological persistence of ametryn, diuron, and oxyfluorfen in soil
}

\author{
Luis Antonio Peñaherrera-Colina², Itamar Ferreira de Souza³ ${ }^{3}$ Luiz Roberto Guimarães Guilherme², \\ Júlio Sílvio de Sousa Bueno Filho ${ }^{3}$
}

\begin{abstract}
RESUMO
Realizou-se este trabalho com o objetivo de avaliar a persistência biológica de herbicidas de pré-emergência em condições de casa-de-vegetação utilizando a aveia (Avena sativa L.) como planta teste. O experimento foi instalado na Estação Experimental Boliche, Província de Guayas, Equador entre outubro e dezembro de 2002. O delineamento experimental foi de blocos ao acaso com quatro repetições, em esquema fatorial $(3 \times 4 \times 4+1)$, envolvendo aplicações de três herbicidas (ametryn a $2,0 \mathrm{~kg} \mathrm{ha}^{-1}$, diuron a $3,2 \mathrm{~kg}$ $\mathrm{ha}^{-1}$ e oxyfluorfen a $0,48 \mathrm{~kg} \mathrm{ha}^{-1}$ ) no solo, mais uma testemunha sem aplicação de herbicida. Após as aplicações este foi amostrado em quatro profundidades (0-5, 5-10, 10-15 e 15-20 cm), em quatro épocas distintas (10, 20, 30 e 40 dias após aplicação). As amostras foram coletadas do campo utilizando-se cilindros de PVC. Nessas amostras cultivou-se aveia para as avaliações de persistência biológica dos produtos. Não se observaram efeitos dos tratamentos na germinação da aveia até os 40 dias após a aplicação (daa). Maior sintoma visual de fitotoxicidade e menor acúmulo de matéria seca total (MST), matéria seca da parte aérea (MAS) e matéria seca da raiz (MSR) foram observadas em plantas de aveia cultivadas em amostras de solo tratadas com diuron coletadas na camada de $0-5 \mathrm{~cm}$ aos 10, 20, 30 e 40 dias. A aveia mostrou alta sensibilidade aos herbicidas testados e desses produtos o diuron foi o que apresentou a maior persistência e a menor mobilidade no solo.
\end{abstract}

Termos para indexaçao: Lixiviação, herbicida, bioensaio.

\begin{abstract}
This investigation had the objective of evaluating the biological persistence of pre-emergence herbicides under greenhouse conditions. Oat plants were used as test organisms. The experiment was established at Boliche Experiment Station, Província Guayas, Ecuador from October to December/2002. The experimental design was a randomized block, in a $3 \times 4 \times 4+1$ factorial scheme, involving three herbicides (ametryn at $2.0 \mathrm{~kg} \mathrm{ha}^{-1}$, diuron at $3.2 \mathrm{~kg} \mathrm{ha}^{-1}$, and oxyfluorfen at $0.48 \mathrm{~kg} \mathrm{ha}^{-1}$ ), plus a control. After herbicide applications soils were sampled in four different depths $(0-5,5-10,10-15$, and $15-20 \mathrm{~cm})$ at four dates $(10,20,30$, and 40 days after herbicide applications). Soil layers were colleted from field using PVC pipes, which were planted with 10 oat seeds. The herbicides did not promote oat seeds emergence reduction. Diuron at the $0-5 \mathrm{~cm}$ layer showed the highest visual symptoms of phytotoxicity and the lowest production of dry matter (total, shoots, and roots) per oat plants at all sampling dates (10, 20, 30, and, 40 days after herbicide applications). Oat plants showed good sensitivity to the herbicide residues. Diuron was more persistent in the soil when compared with ametryn and oxyfluorfen.
\end{abstract}

Index terms: Leaching, herbicide, bioassay.

(Recebido para publicação em 7 de maio de 2003 e aprovado em 4 de maio de 2005)

\section{INTRODUÇÃO}

Os herbicidas são hoje uma importante ferramenta para o manejo das plantas daninhas no sistema de produção intensivo de diferentes culturas. Uma quantidade considerável de herbicida é aplicada diretamente ao solo. Outros, embora aplicados sobre as plantas daninhas, acabam chegando ao solo direta ou indiretamente, podendo ser absorvidos pelas plantas, lixiviado e incorporado ao lençol freático, ou sofrer degradação. Paralelamente à degradação, o herbicida acumula-se no ambiente, tornando-se fundamental seu uso adequado tanto para preservar a qualidade dos produtos colhidos quanto os recursos que sustentam a produção.

Quando aplicados ao solo, ficam retidos na camada superior por um tempo que está em função do teor de argila e/ou matéria orgânica, e das características físico-químicas do produto (ESALQ, 2002).

O movimento de um herbicida depende basicamente das interações entre sua estrutura molecular e as características do solo (textura, estrutura, M.O., pH, CTC) e de manejo a qual a área é submetida, além dos fatores climáticos (pluviosidade, temperatura, etc) (ESALQ, 2002).

${ }^{1}$ Parte de Tese de Mestrado apresentada pelo primeiro autor à Universidade Federal de Lavras. Apresentado para publicação em abril de 2003.

${ }^{2}$ Agrônomo, MS - Departamento de Agricultura, Universidade Federal de Lavras/UFLA - Cx. P. 3037 - 37.200-000 - Lavras, MG.

${ }^{3}$ Agrônomo, DS - Departamento de Agricultura/UFLA.

${ }^{3}$ Agrônomo, DS - Departamento de Ciência do Solo/UFLA.

${ }^{3}$ Agrônomo, DS - Departamento de Ciencias Exatas/UFLA. 
Esta dinâmica é governada por alguns fenômenos denominados: retenção (habilidade de reter molécula orgânica evitando sua saída da matriz); adsorção ou sorção (processo reversível de retenção e atração de molécula química na superfície de colóide por tempo dependente de sua afinidade) (ESALQ, 2002).

Pesquisas desenvolvidas por Ahmad et al. (2001) com diferentes solos do Paquistão (valores baixos de Kd) e da Austrália, concluíram que o pH e o carbono orgânico apresentaram alta correlação com a sorção do ametryn em solos com características alcalinas. Para imazethapyr o pH foi o fator mais importante. Porém, estes herbicidas apresentam alto risco de contaminação das águas subterrâneas.

Yen et al. (2003) estudaram a dissipação e mobilidade do oxyfluorfen em diferentes solos sob diferentes condições de umidade e temperatura e concluíram que altas temperaturas aumentaram a taxa de dissipação. A possibilidade de contaminação de água subterrânea por oxyfluorfen é muito baixa e só é possível quando o solo apresenta níveis muito baixos de matéria orgânica.

O bioensaio é uma das formas empregadas para estudar atividade, persistência e mobilidade do químico aplicado, por ser simples, bastante sensível, rápido e de baixo custo. Também não requer equipamento sofisticado comparado com métodos analíticos
(SANTELMAN, 1977).

O presente trabalho teve como objetivo identificar a persistência dos herbicidas ametryn, diuron e oxyfluorfen aplicados em dois tipos de solos utilizando bioensaios com aveia branca como planta teste.

\section{MATERIAL E MÉTODOS}

O experimento foi conduzido no ano de 2002 entre os meses de outubro e dezembro em casa-de-vegetação na Estação Experimental Boliche, INIAP no km 26 da rodovia Durán-Tambo, Equador, a $17 \mathrm{~m}$ altitude, $0^{\circ} 15^{\circ}$ de latitude Sul e $79^{\circ} 58^{\prime}$ longitude Oeste. As variações de temperatura do ar ocorridas durante o desenvolvimento do experimento foram obtidas na Estação Experimental Boliche (Figura 1). O solo usado no experimento foi classificado como INSEPTISOL NUEVO (BUOL et al., 1990) de textura média. Os resultados da análise química das amostras são apresentados na Tabela 1.

Utilizou-se um delineamento de blocos casualizados, com 4 repetições. Os tratamentos foram dispostos em esquema fatorial $3 \times 4 \times 4+1$, sendo os fatores: três herbicidas (ametryn- Gesapax $80 \mathrm{PM}$ a 2,0 $\mathrm{kg} \mathrm{ha}^{-1}$, diuronKarmex 80 WG a 3,2 kg ha ${ }^{-1}$, oxyfluorfen-Goal 240 CE a 0,48 $\left.\mathrm{kg} \mathrm{ha}^{-1}\right)$, quatro épocas de coleta do solo $(10,20,30$ e 40 dias após da aplicação), quatro camadas de solo coletadas $(0-5,5-10,10-15$ e $15-20 \mathrm{~cm})$ mais uma testemunha sem herbicida.

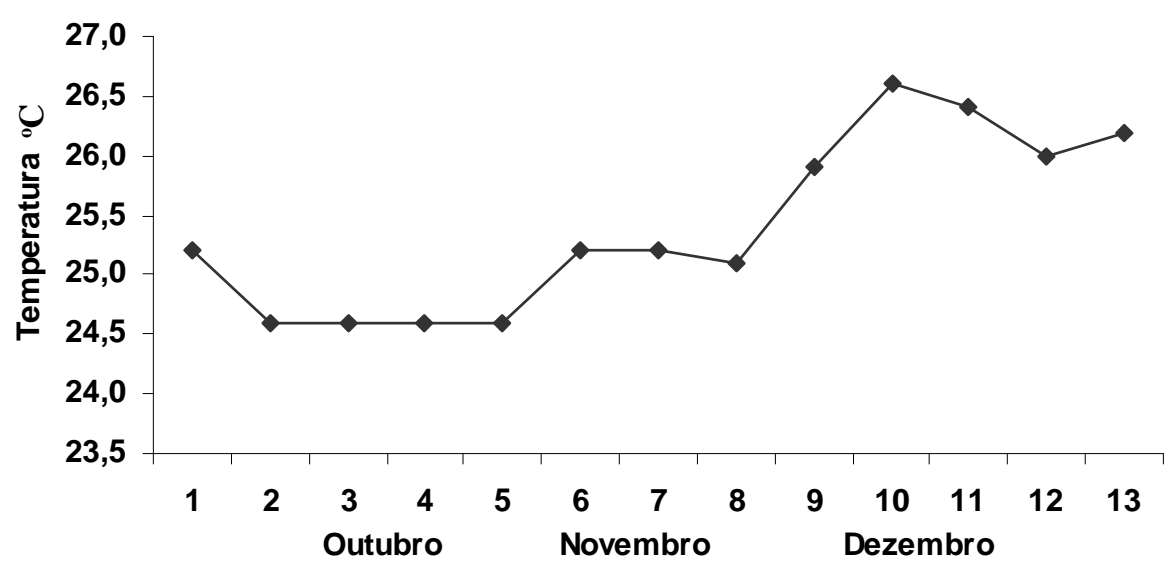

FIGURA 1 - Variação da temperatura de outubro a dezembro/2002 em Boliche, Equador. 2002. 
TABELA 1 - Atributos químicos e físicos de amostras de solos utilizados nos experimentos em Boliche. Equador. 2002.*

\begin{tabular}{|c|c|c|c|}
\hline \multicolumn{2}{|c|}{ Características } & \multicolumn{2}{|c|}{ Boliche } \\
\hline Atributos & Unidades & $0-10 \mathrm{~cm}$ & $10-20 \mathrm{~cm}$ \\
\hline \multicolumn{4}{|l|}{ Químicas } \\
\hline pH (água) & & 5,8 & 6,0 \\
\hline $\mathrm{P}$ & $\mathrm{mg} \mathrm{dm}^{-3}$ & 28 & 26 \\
\hline $\mathrm{K}$ & $\mathrm{mg} \mathrm{dm}^{-3}$ & 195,5 & 172,0 \\
\hline $\mathrm{Ca}$ & $\mathrm{cmol}_{\mathrm{c}} \mathrm{dm}^{-3}$ & 13,0 & 16,0 \\
\hline $\mathrm{Mg}$ & $\mathrm{cmol}_{\mathrm{c}} \mathrm{dm}^{-3}$ & 3,5 & 5,9 \\
\hline $\mathrm{Al}+\mathrm{H}$ & $\mathrm{cmol}_{\mathrm{c}} \mathrm{dm}^{-3}$ & 0,10 & 0,15 \\
\hline Matéria orgânica & dag $\mathrm{kg}^{-1}$ & 1,8 & 1,4 \\
\hline CTC & $\mathrm{cmol}_{\mathrm{c}} \mathrm{dm}^{-3}$ & 17,1 & 22,4 \\
\hline \multicolumn{4}{|l|}{ Físicas } \\
\hline Areia & $\mathrm{g} \mathrm{kg}^{-1}$ & 260 & 300 \\
\hline Silte & $\mathrm{g} \mathrm{kg}^{-1}$ & 520 & 460 \\
\hline Argila & $\mathrm{g} \mathrm{kg}^{-1}$ & 220 & 240 \\
\hline Textura & & Fco-limoso & Franco \\
\hline
\end{tabular}

* Análises realizadas no Laboratório do Departamento de Solos e Águas da Estación Experimental Boliche do INIAP. Equador. 2002

Cada unidade experimental constituiu-se de um cilindro de PVC de $100 \mathrm{~mm}$ de diâmetro por $25 \mathrm{~cm}$ de comprimento, que foi preenchido com material de solo agrícola indeformado. Na extremidade inferior foi colocado um funil plástico e tela metálica com papel de filtro Whatman $\mathrm{n}^{\circ} 400$ para evitar a perda do material do solo. Os cilindros foram colocados numa bancada em posição vertical para aplicação de solução de $\mathrm{CaCl}_{2} 0,01 \mathrm{~mol} \mathrm{~L}^{-1}$ na quantidade de $1000 \mathrm{~mL}$ dia com propósito de estabilizar iônicamente, homogeneizar o $\mathrm{pH}$ e devolver as características de estrutura ao solo que possa ter sido alterada no momento da coleta. Este tratamento permaneceu por um período de 10 dias. Após esse período os herbicidas foram aplicados na extremidade aberta (superior) do cilindro num volume de $0,36 \mathrm{~mL}$ equivalente a $400 \mathrm{~L} \mathrm{ha}^{-1}$, aplicando o correspondente a 2,0 L, 3,2 L e 0,48 $\mathrm{L} \mathrm{ha}^{-1}$ de ametryn, diuron e oxyfluorfen, respectivamente, distribuindo o produto de maneira uniforme na superfície do solo.

Após 24 horas da aplicação dos herbicidas nos cilindros, foi simulada chuva com lâmina de $6 \mathrm{~mm}$ por dia, durante a permanência dos cilindros na bancada, segundo histórico de precipitação da região no verão.

Em cada época de amostragem o cilindro foi tirado da bancada e o solo separado em camadas a cada $5 \mathrm{~cm}$ e colocado num pote plástico com capacidade de $500 \mathrm{~mL}$, colocando nele 10 sementes da planta indicadora. Os potes foram mantidos em casa-de-vegetação por 17 dias e a matéria seca total (MST), a parte aérea (MSA) e a parte radicular (MSR) foram avaliadas no final deste período.

A fitotoxicidade ( 0 a $100 \%$ dano) sobre plântulas de aveia causada aos 14 dias após a semeadura nos cilindros foi avaliada. Aos 17 dias as plantas foram colhidas e secas em estufa de circulação forçada de ar à temperatura de $60^{\circ} \mathrm{C}$ por um período de 72 horas. Após a secagem, foi realizada a pesagem para obtenção da MST, MAS e MSR.

Na casa-de-vegetação, a umidade do solo foi mantida próxima da capacidade de campo $(80 \%)$ procurando mantê-la neste nível durante a fase de condução do experimento. Para isso, foi utilizado o método gravimétrico, pesando-se diariamente os vasos e recompondo seu peso pela irrigação. Aos 12 dias foi feita uma irrigação com solução nitrogenada visando crescimento normal das plantas.

Os dados obtidos foram submetidos à análise de variância, aplicando-se o teste de $\mathrm{F}$ e desdobrando as interações significativas com ajuda do software 'SISVAR'. Para melhor observar os efeitos dos tratamentos, utilizou-se superfície de resposta na apresentação de gráficos para a interação tripla.

\section{RESULTADOS E DISCUSSÃO}

\section{Fitotoxicidade}

A análise das notas de fitotoxicidade observada aos 14 dias após a semeadura (Figura 2) indica efeito de 
herbicidas nas diferentes épocas de amostragem. O ametryn (H1) provocou dano inicial pequeno com recuperação das plantas. Para diuron $(\mathrm{H} 2)$, a toxicidade às plantas de aveia foi evidente em todas as épocas de plantio com morte das plantas, mesmo com tendência de queda ao longo do tempo coincidindo com o mencionado pela WSSA (1994), que considera o diuron como medianamente persistente, mas dependendo da umidade e do tipo do solo. O oxyfluorfen (H3) não proporcionou sintomas fitotóxicos na planta indicadora em nenhuma época de plantio, provavelmente, devido a sua rápida transformação ou inativação no solo (WSSA, 1994).

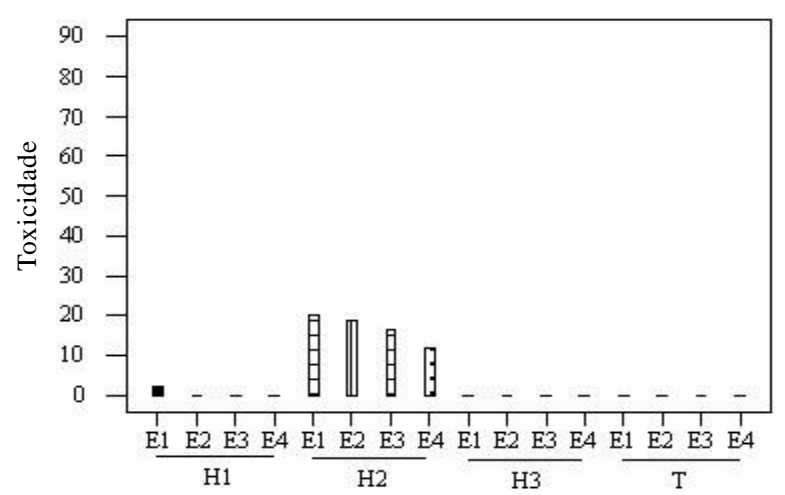

FIGURA 2 - Toxicidade em plantas de aveia plantada em quatro épocas $(E 1=10$ daa, E2=20 daa, E3=30 daa, E4=40 daa) após a aplicação de quatro herbicidas (H1=ametryn, $\mathrm{H} 2=$ diuron, $\mathrm{H} 3=$ oxyfluorfen, $\mathrm{T}=$ sem herbicida). E.E. Boliche. Equador. 2002.

\section{Matéria seca}

Houve interação tripla para a matéria seca total, da parte aérea e do sistema radicular das plantas de aveia entre diferentes tratamentos. Dessa forma, o efeito de cada herbicida foi considerado separadamente.

\section{Matéria seca total (MST)}

O herbicida ametryn (Figura 3, A) não provocou redução na MST de plantas de aveia cultivadas nas amostras de solos feitas ao longo do tempo, até aos 40 dias.

O diuron (Figura 3, D) reduziu a MST de plantas cultivadas em amostras de solo coletadas na camada de 0 $5 \mathrm{~cm}$, em todas as épocas de amostragem, demonstrando pouco potencial de lixiviação. Resultados semelhantes foram encontrados por Mussini et al. (1995), que avaliaram a lixiviação do diuron em solos com diferentes texturas. Ao contrário, Victoria Filho et al. (1991), em avaliação na produção de citros detectaram que o diuron e outros herbicidas em estudo aplicados ao longo de 15 anos tiveram influência mínima sobre a cultura, apesar de haver pequena variância em alguns anos.

Para o herbicida oxyfluorfen (Figura 3, O) foi observado um efeito não-significativo na redução de MST da aveia, quando utilizado em amostras de solos colhidos aos 10 dias e $20 \mathrm{~cm}$ de profundidade e aos 40 dias nas profundidades de 5-10 e 10-15 cm. Em testes feitos por Souza et al. (1996), destacaram-se a variação da microbiota de solos tratados com oxyfluorfen e outros herbicidas, devido as características físicas do solo.

\section{Matéria seca da parte aérea (MSA)}

O ametryn (Figura 4, A), não afetou a produção de MSA nas diferentes épocas de avaliação.

O diuron (Figura 4, D) proporcionou redução acentuada na produção de MSA da aveia aos 10, 20, 30 e 40 dias após a aplicação do produto, nas amostras colhidas a 0-5 cm de profundidade, confirmando suas características de pouca mobilidade nas condições testadas. Esses resultados indicam que o herbicida apesar de pouco móvel, permaneceu disponível (adsorção fraca).

\section{Matéria seca da raiz (MSR)}

Na matéria seca da raiz também foram observados diferenças entre os tratamentos, seguindo a tendência apresentada nas variáveis estudadas anteriormente. $\mathrm{O}$ ametryn (Figura 6, A) apresentou efeito negativo na MSR quando a amostragem foi feita aos 10 dias. Isto foi reflexo da fitotoxicidade observada, que pode ter reduzido a taxa de crescimento da planta no período de estresse, a qual posteriormente retomou o crescimento normal da planta.

Para o caso do diuron (Figura 5, D), a MSR da aveia foi severamente afetada apresentando queda em todas as épocas de amostragem nos primeiros $5 \mathrm{~cm}$ de profundidade no solo. Paulo et al. (1991) ressaltam a importância da CTC e classe textural dos solos para a avaliação de resíduo de diuron nas raízes das plantas, e em solos com pouca matéria orgânica onde o processo de degradação acontece ainda mais lentamente (OLIVEIRA JÚNIOR, 2002).

No caso do oxyfluorfen (Figura 5, O), a MSR foi pouco afetada, resultado da baixa toxicidade observada. Em épocas tardias, a perda de vigor do material de plantio pode explicar a menor taxa de produção de matéria seca. 
A

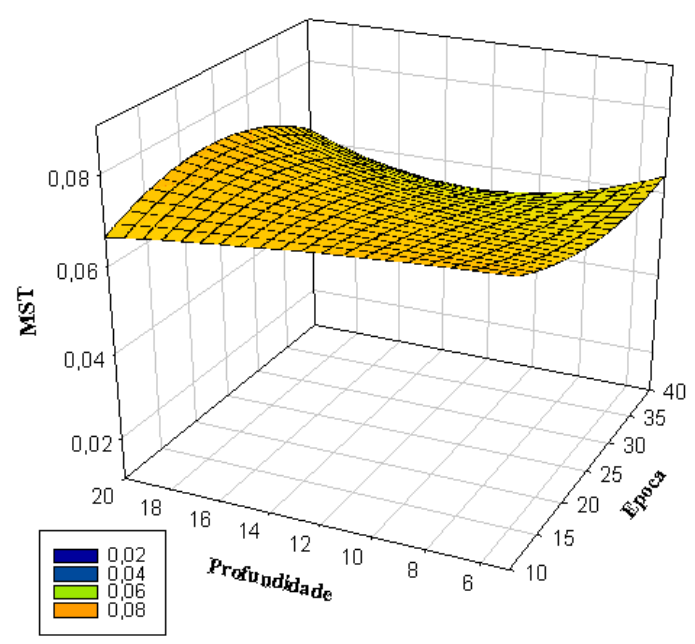

$\hat{\mathrm{Y}}=0,0918-0,0022 \mathrm{e}+4,93 \mathrm{E}-5 \mathrm{e}^{2}-0,00137 \mathrm{p}-2,93 \mathrm{E}-$ $5 p^{2}+1,97 E-4 e p-6,7 E-6 e^{2} p+1,36 E-7 e p^{2}+1,18 E-7 e^{2} p^{2}$ $\mathbf{R}^{2}=\mathbf{0 , 5 8}$
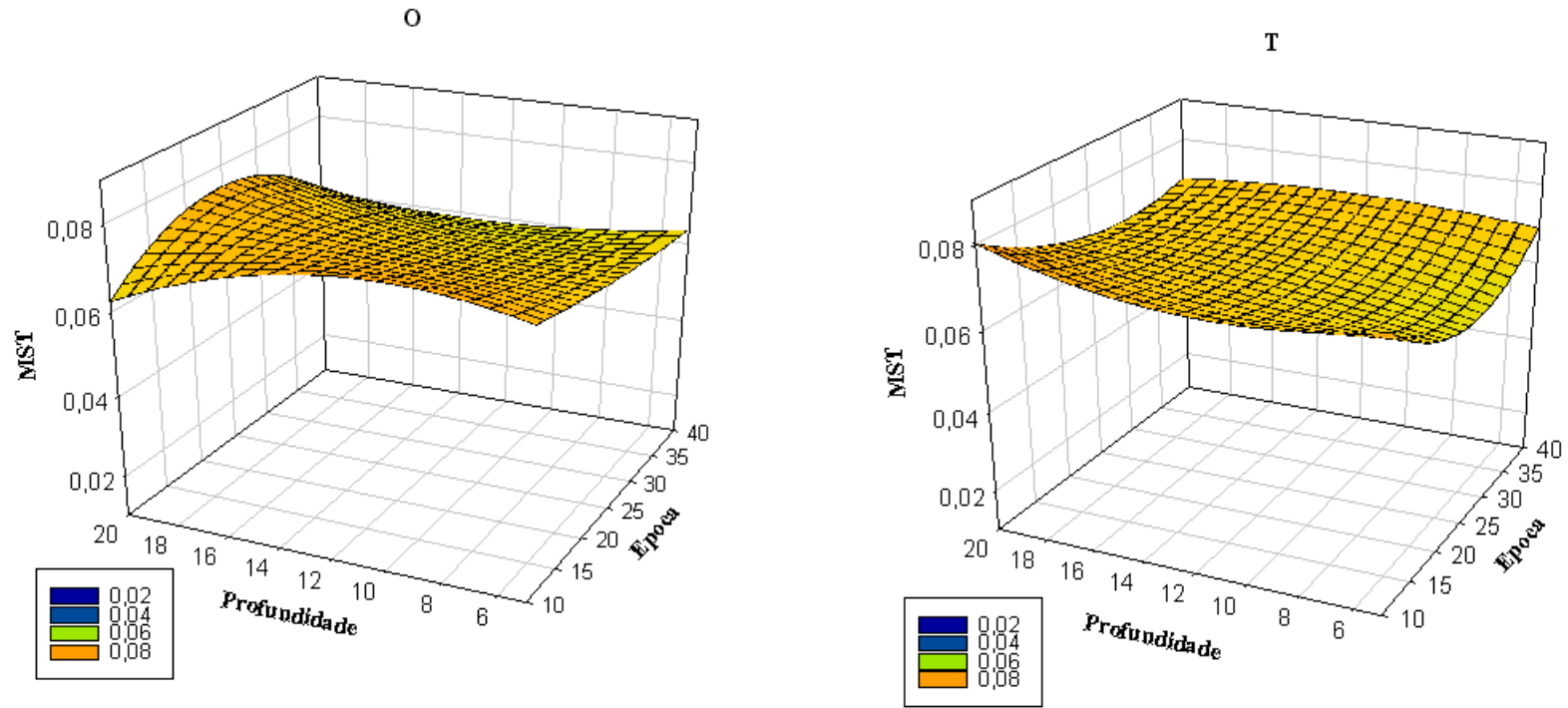

\footnotetext{
$\hat{\mathrm{Y}}=0,0528+0,00107 \mathrm{e}-1,59 \mathrm{E}-5 \mathrm{e}^{2}+0,0070 \mathrm{p}-3,88 \mathrm{E}-4 \mathrm{p}^{2}$. $4,77 \mathrm{E}-4 \mathrm{ep}+6,48 \mathrm{E}-6 \mathrm{e}^{2} \mathrm{p}+2,84 \mathrm{E}-5 \mathrm{ep}^{2}-4,3 \mathrm{E}-7 \mathrm{e}^{2} \mathrm{p}^{2}$ $\mathbf{R}^{2}=\mathbf{0 , 5 6}$
}

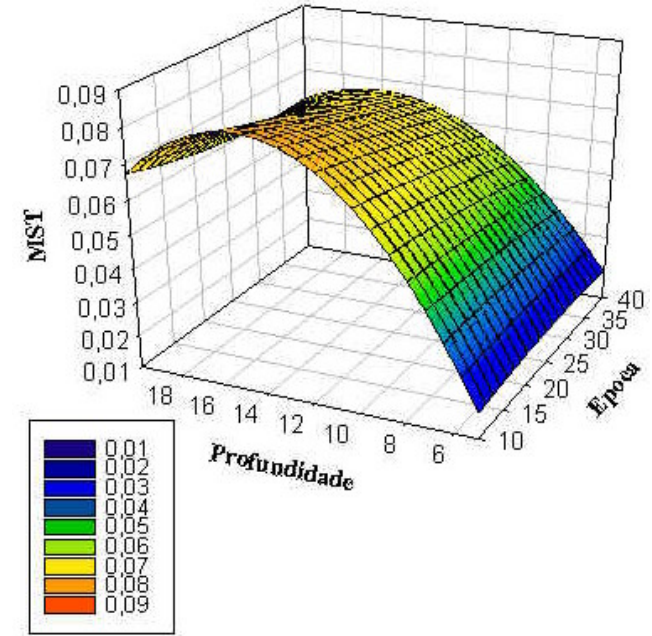

$\hat{\mathrm{Y}}=-0,0767+0,00132 \mathrm{e}-6,56 \mathrm{E}-6 \mathrm{e}^{2}+0,0216 \mathrm{p}-7,04 \mathrm{E}-4 \mathrm{p}^{2}-$ 2,07E-4ep-5,12E-7e $\mathrm{e}^{2} \mathrm{p}+4,84 \mathrm{E}-6 \mathrm{e}^{2}+7,5 \mathrm{E}-8 \mathrm{e}^{2} \mathrm{p}^{2}$ $\mathbf{R}^{2}=0,92$

\footnotetext{
$\hat{\mathrm{Y}}=0,129-0,00543 \mathrm{e}+9,5 \mathrm{E}-5 \mathrm{e}^{2}-0,00757 \mathrm{p}+2,95 \mathrm{E}-$ $4 p^{2}+6,1 \mathrm{E}-4 \mathrm{ep}-9,86 \mathrm{E}-6 \mathrm{e}^{2} \mathrm{p}-2,17 \mathrm{E}-5 \mathrm{ep}^{2}+3,32 \mathrm{E}-7 \mathrm{e}^{2} \mathrm{p}^{2}$ $\mathbf{R}^{2}=\mathbf{0 , 4 7}$
}

FIGURA 3 - Matéria seca total (MST) da aveia tratada com ametryn (A), diuron (D), oxyfluorfen (O) e testemunha (T) em diferentes épocas de plantio (10, 20, 30 e 40 dias após a aplicação) e várias profundidades $(0-5-\mathrm{cm}, 5-10 \mathrm{~cm}, 10-15 \mathrm{~cm}$ e 15-20 cm), E.E. Boliche, Equador. Lavras, 2002.

Ciênc. agrotec., Lavras, v. 29, n. 5, p. 980-987, set./out., 2005 
A

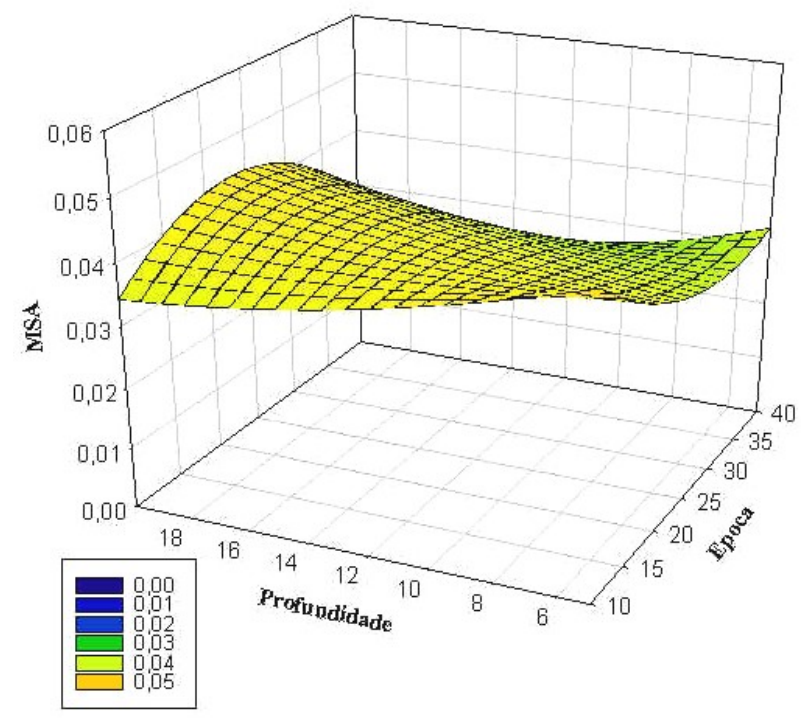

$\hat{\mathbf{Y}}=0,092-0,00435 \mathrm{e}+7,86 \mathrm{E}-5 \mathrm{e}^{2}-0,00609 \mathrm{p}+1,1 \mathrm{E}-4 \mathrm{p}^{2}+$ $5,26 \mathrm{E}-4 \mathrm{ep}-1,1 \mathrm{E}-5 \mathrm{e}^{2} \mathrm{p}-9,17 \mathrm{E}-6 \mathrm{ep}^{2}+2,19 \mathrm{E}-7 \mathrm{e}^{2} \mathrm{p}^{2}$ $\mathbf{R}^{2}=\mathbf{0 , 6 1}$

$\mathrm{O}$

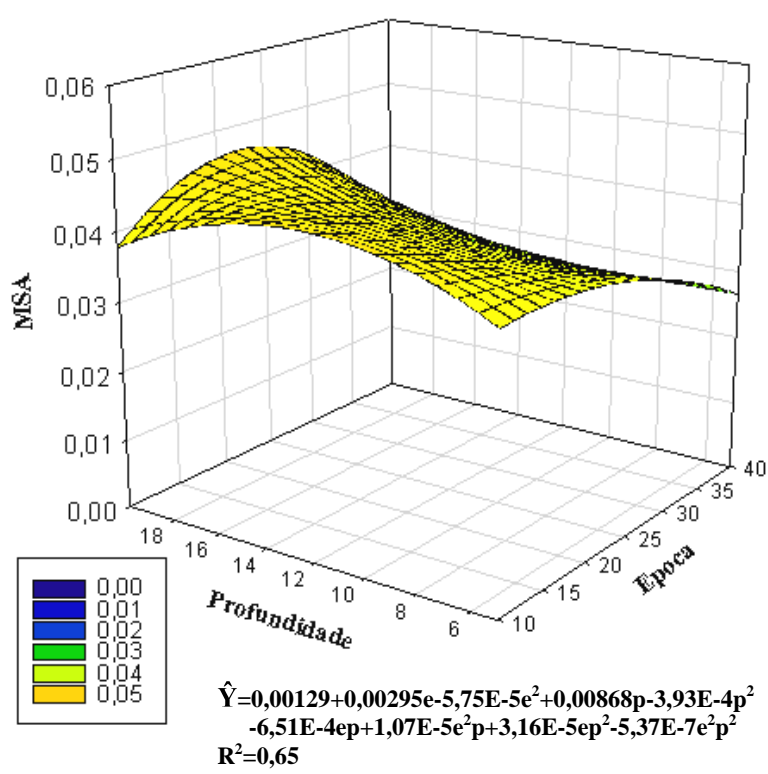

$\mathrm{D}$

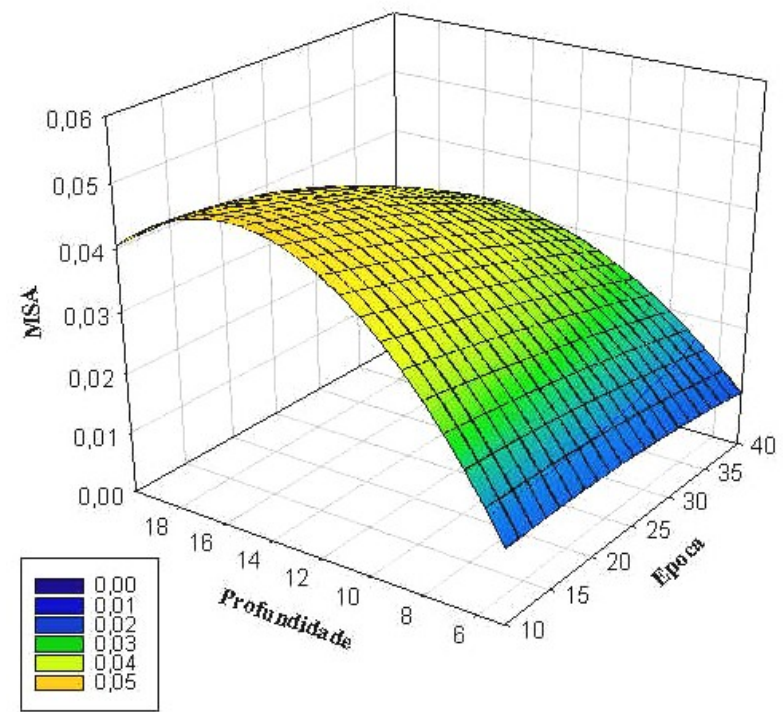

$\hat{\mathrm{Y}}=-0,0443+0,00129 \mathrm{e}-1,51 \mathrm{E}-5 \mathrm{e}^{2}+0,0133 \mathrm{p}-4,61 \mathrm{E}-4 \mathrm{p}^{2}-$ $2,82 \mathrm{E}-4 \mathrm{ep}+2,56 \mathrm{E}-6 \mathrm{e}^{2} \mathrm{p}+1,2 \mathrm{E}-5 \mathrm{ep}^{2}-1,34 \mathrm{E}-7 \mathrm{e}^{2} \mathrm{p}^{2}$ $R^{2}=0,90$

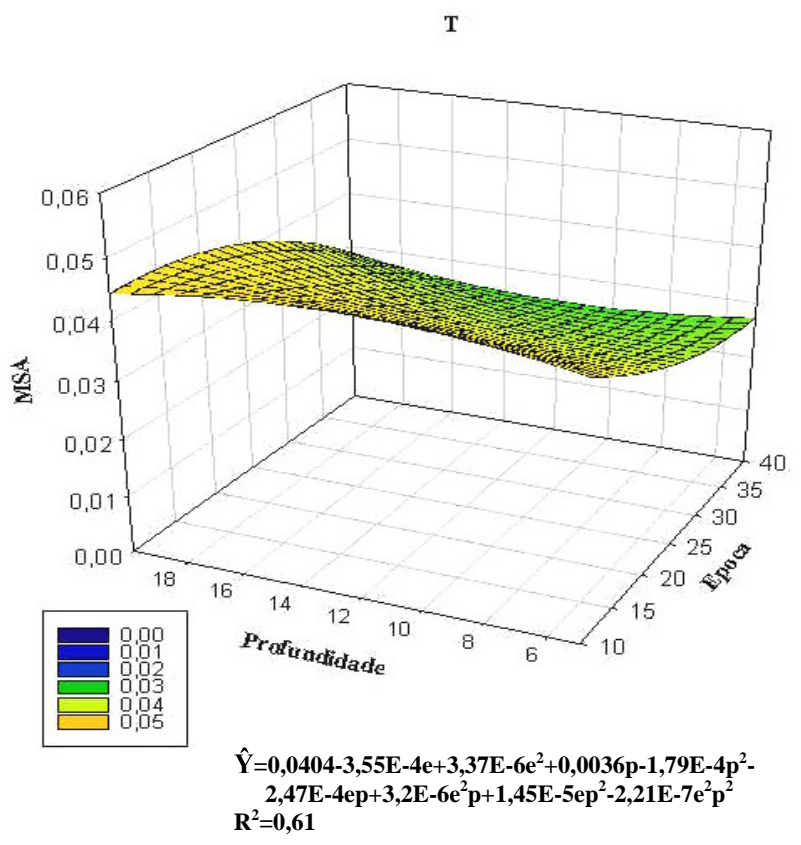

FIGURA 4 - Matéria seca parte aérea (MSA) de aveia tratada com ametryn (A), diuron (D), oxyfluorfen (O) e testemunha (T) em varias épocas (10, 20, 30 e 40 dias após a aplicação) e diferentes profundidades $(0-5 \mathrm{~cm}, 5-10 \mathrm{~cm}, 10-15 \mathrm{~cm}$ e 15 $20 \mathrm{~cm}$ ), E.E. Boliche, Equador. 2002.

Ciênc. agrotec., Lavras, v. 29, n. 5, p. 980-987, set./out., 2005 
A

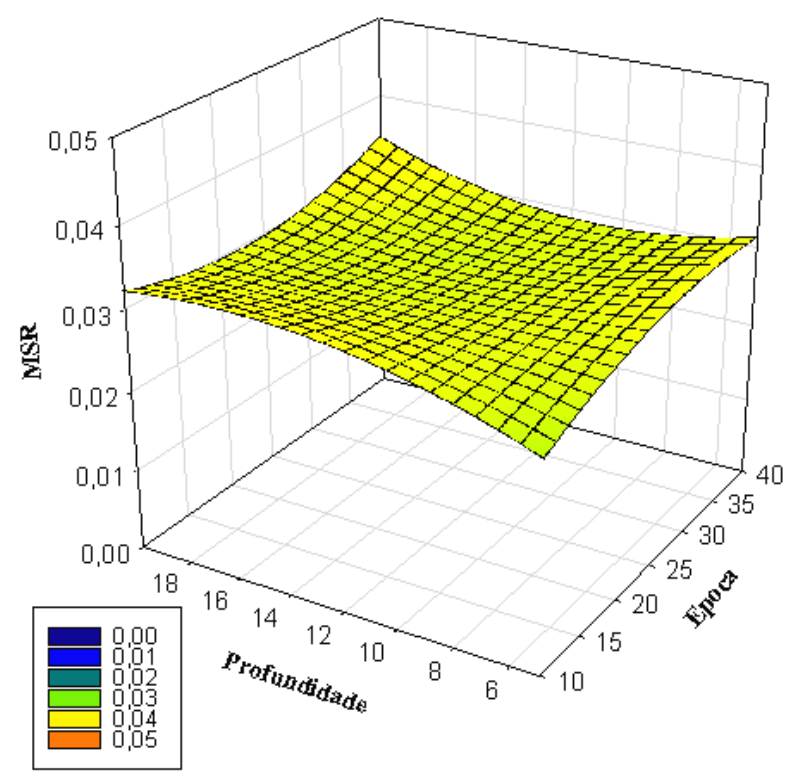

$\hat{\mathbf{Y}}=-4,33 \mathrm{E}-4+0,0021 \mathrm{e}-2,93 \mathrm{E}-5 \mathrm{e}^{2}+0,00472 \mathrm{p}-1,4 \mathrm{E}-4 \mathrm{p}^{2}-$ $3,29 \mathrm{E}-4 \mathrm{ep}+4,3 \mathrm{E}-6 \mathrm{e}^{2} \mathrm{p}+9,31 \mathrm{E}-5 \mathrm{ep}^{2}-1,01 \mathrm{E}-7 \mathrm{e}^{2} \mathrm{p}^{2}$ $\mathbf{R}^{2}=\mathbf{0 , 7 7}$

o

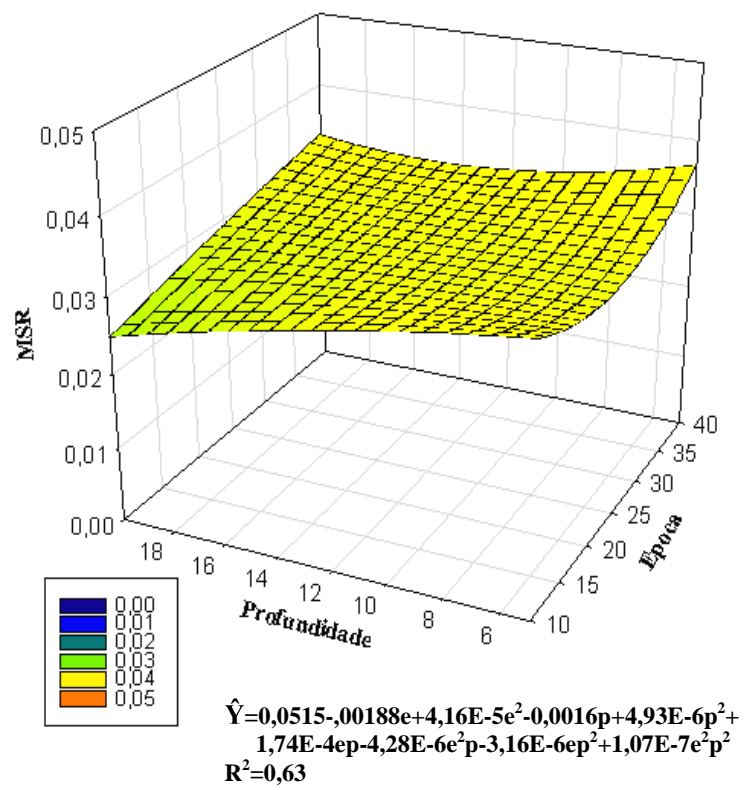

D

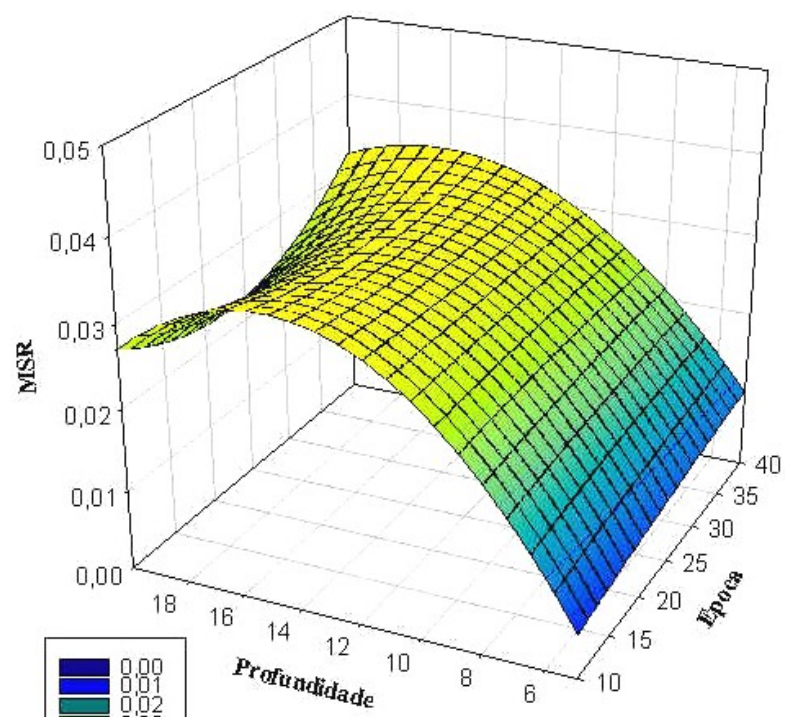

$\hat{\mathrm{Y}}=-0,0324+3,02 \mathrm{E}-5 \mathrm{e}+8,57 \mathrm{E}-6 \mathrm{e}^{2}+0,00834 \mathrm{p}-2,42 \mathrm{E}-4 \mathrm{p}^{2}$ $+7,46 \mathrm{E}-5$ ep-307E-6e $2 \mathrm{p}-7,21 \mathrm{E}-6 \mathrm{ep}^{2}+2,09 \mathrm{E}-7 \mathrm{e}^{2} \mathrm{p}^{2}$ $R^{2}=0,89$

$\mathrm{T}$

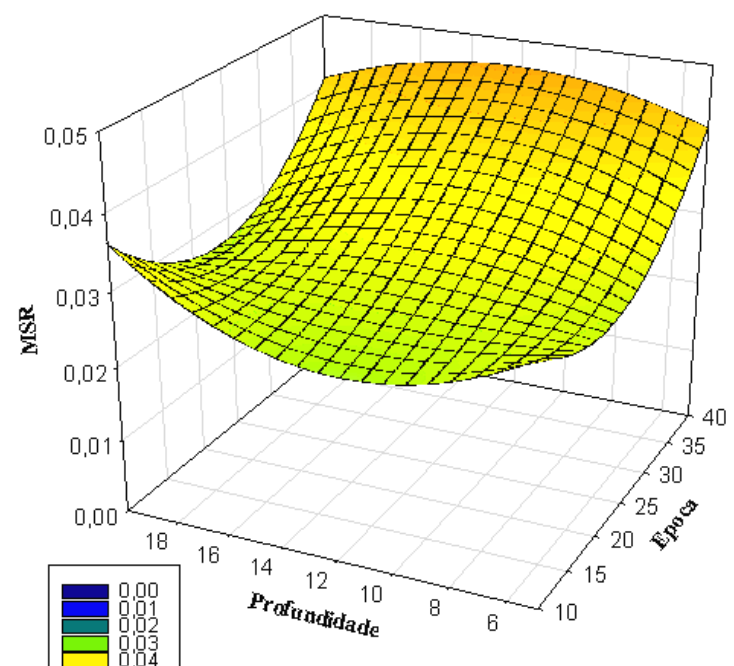

$\hat{\mathrm{Y}}=0,089-0,00507 \mathrm{e}+9,16 \mathrm{E}-5 \mathrm{e}^{2}-0,011 \mathrm{p}+4,74 \mathrm{E}-4 \mathrm{p}^{2}+$ 8,58E-4ep-1,30E-5e $\mathrm{e}^{2}-3,62 \mathrm{E}-5 \mathrm{ep}^{2}+5,54 \mathrm{E}-7 \mathrm{e}^{2} \mathrm{p}^{2}$ $\mathbf{R}=\mathbf{0 , 8 6}$

FIGURA 5 - Matéria seca da raiz (MSR) de aveia tratada com ametryn (A), diuron (D) e oxyfluorfen (O) e testemunha (T) em várias épocas (10, 20, 30 e 40 dias após a aplicação) e diferentes profundidades $(0-5 \mathrm{~cm}, 5-10 \mathrm{~cm}, 10-15 \mathrm{~cm}$ e 15 $20 \mathrm{~cm}$ ), E.E. Boliche, Equador. 2002.

Ciênc. agrotec., Lavras, v. 29, n. 5, p. 980-987, set./out., 2005 


\section{CONCLUSÕES}

A aveia mostrou alta sensibilidade aos herbicidas avaliados e desses produtos, o diuron foi o que apresentou a maior persistência e a menor mobilidade do solo.

\section{REFERÊNCIAS BIBLIOGRÁFICAS}

AHMAD, R.; KOOKANA, R. S.; ALSTON, A. M. Sorption of ametryn and imazethapyr in twenty-five soils from Pakistan and Australia. Journal of Environmental Science and Health Part B-Pesticides Food Contaminants and Agricultural Wastes, New York, v. 36, n. 2, p. 143-160, 2001.

BUOL, S. W.; HOLE, F. D.; McCRACKEN, J. R. Génesis y clasificación de suelos. 2. ed. México: Trillas, 1990. 417 p.

ESCOLA SUPERIOR DE AGRICULTURA LUIZ DE QUEIROZ. Dinâmica de defensivos agrícolas no solo: aspectos práticos e ambientais. Piracicaba, 2002. 96 p. Apostila.

MUSSINI, M. R.; NAKAGAMA, L. E.; LUCHINI, L. C.; MATALLO, M. B.; ANDREA, M. M. Degradação do diuron 14C em solos e planta de cana de açúcar (Sarcharum spp.). Pesquisa Agropecuária Brasileira, Brasília, v. 30, n. 6, p. 775-778, jun. 1995.

OLIVEIRA JÚNIOR, R. S. Comportamento de herbicidas nos solos do Brasil. In: REUNIÃO DE PESQUISADORES EM CONTROLE DE PLANTAS DANINHAS, 14., 2002,
Goiânia. Resumos... Goiânia: Tarcisio Cobucci, 2002. p. 27-57.

PAULO, E. M.; JORGE, J. A.; ARRUDA, F. B.; PATRICIO, F. R. A. Efeitos de algumas características de solo na resposta da planta a dose de herbicida. Planta Daninha, Rio de Janeiro, v. 9, n. 1/2, p. 76-84, 1991.

SANTELMAN, P. W. Herbicide bioassay. In: TRULOVE, B. (Ed.). Research method in weed science. 2. ed. [S.1.]: Southern Weed Science Society, 1977. p. 79-87.

SOUZA, A. P. de; LOURES, E. G.; SILVA, F. J. da; RUIZ, H. A. Efeito do oxyfluorfen, 2,4-D e glyphosate na atividade microbiana de solos com diferentes texturas e conteúdo de matéria orgânica. Planta Daninha, Rio de Janeiro, v. 14, n. 1, p. 55-64, 1996.

VICTORIA FILHO, R.; MOREIRA, C. S.; SHIMOAMA, N. Y.; SHINOHARA, R. K. Uso contínuo de herbicida em Citros (Citrus sinensis L. Osbedek), efeitos no desenvolvimento, produção e qualidade dos frutos. Planta Daninha, Rio de Janeiro, v. 9, n. 1/2, p. 102-113, 1991.

WEED SCIENTE SOCIETY OF AMERICA. Herbicide handbook. 7. ed. Ahrens, 1994. 352 p.

YEN, J. H.; SHEU, W. S.; WANG, Y. S. Dissipation of the herbicide oxifluorfen in subtrolical soils and its potential to contaminate groundwater. Journal of Ecotoxicology and Environmental Safety, New York, v. 54, n. 2, p. 151-156, Feb. 2003. 